\title{
X-ray broad band spectral Properties of Intermediate Polars
}

\author{
Giorgio Matt
}

Dipartimento di Fisica, Università degli Studi Roma Tre, Via della Vasca Navale 84, I-00146 Roma, Italy

Domitilla de Martino

INAF-Osservatorio Astronomico di Capodimonte, via Moiariello 16, I-80131 Napoli, Italy

Tomaso Belloni

INAF-Osservatorio Astronomico di Brera, Via E. Bianchi 46, I-23807 Merate, Italy

Lucio Chiappetti

IASF-CNR, Sezione di Milano, Via Bassini 15, Milano I-20133, Italy

Frank Haberl

Max-Planck-Institut für extraterrestrische Physik, Giessenbachstrasse, Postfach 1312, 85741 Garching, Germany

\begin{abstract}
We summarize the broad band spectral properties of a small sample of bright Intermediate Polars studied with BeppoSAX.
\end{abstract}

\section{Broad band spectral properties of Intermediate Polars}

Intermediate Polars (IPs) have the hardest X-ray spectra among Magnetic Cataclysmic Variables, with temperatures of several tens of $\mathrm{keV}$. It is therefore very important to observe them in hard X-rays, in order to measure the temperature and estimate the Compton reflection component from the WD surface (Matt 1999). The ROSAT discovery of black body emission in some sources of this class (Haberl \& Motch 1995, and references therein), on the other hand, implies that soft $\mathrm{X}$-ray observations are also necessary to completely characterize their X-ray emission. BeppoSAX, thanks to its broad band, has been unique in offering the possibility to simultaneously measure the temporal and spectral properties of the two components.

BeppoSAX observations of four bright IPs are presented in this contribution. In Table 1 the log of the observations, as well as the spectral results from the phase-averaged spectra, are reported. (The temporal properties, both periodic and non-periodic, cannot be presented here for lack of space, and will be presented in a forthcoming paper). The BeppoSAX observations have detected 
hard X-ray emission up to $90 \mathrm{keV}$ in all sources (apart from RX J1712.6-2414, whose PDS spectrum could not be used due to confusion by a bright cluster of galaxies), providing temperatures of the hard X-ray emission and the Compton reflection component. The data, however, are not of enough quality to derive the multi-temperature structure of the post-shock region. While RE $0751+14$ and RX J0558.0+5353 were discovered as the first soft X-ray IPs, also RX J1712.62414 displays in these BeppoSAX data a hot black-body component thus confirming the recent $X M M$-Newton results found by Haberl (2002). On the other hand, RX J0028 +5917 represents a typical hard X-ray emitting IP.

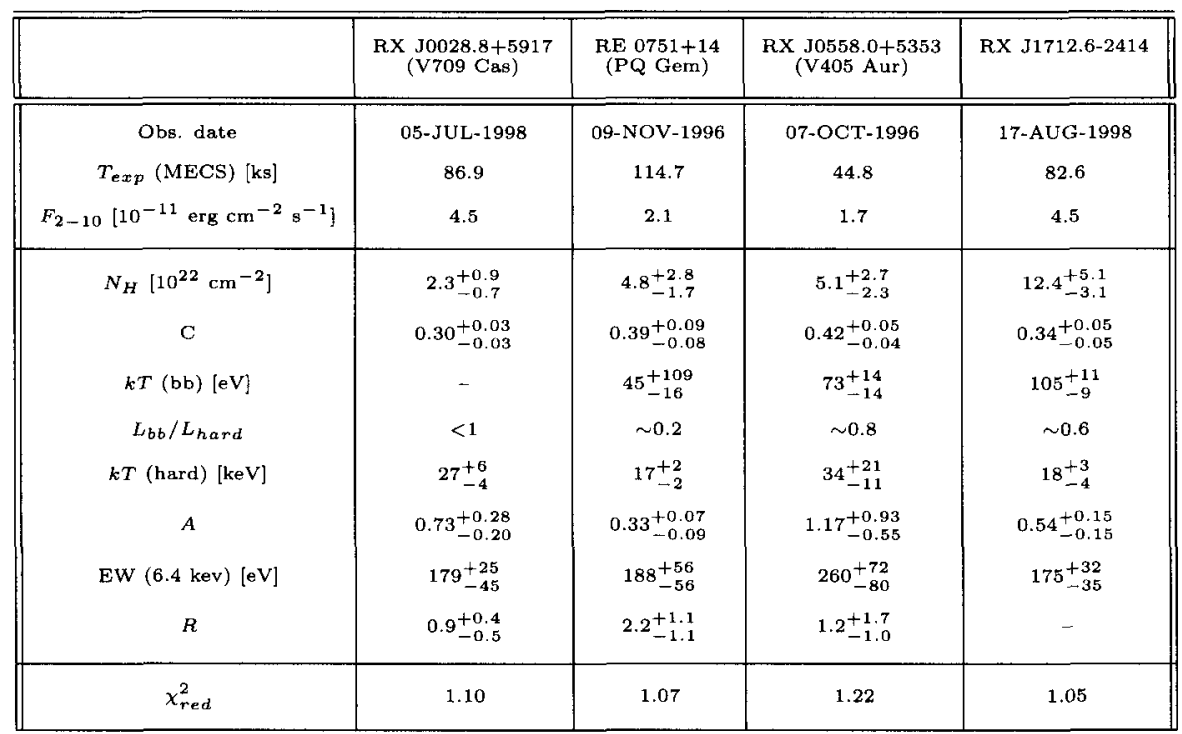

Table 1. Log of the observations and spectral results. The spectral model consists of: thermal plasma emission MEKAL with temperature $k T$ (hard) and metal abundance $A$ (in number with respect to solar); a black body component with temperature $k T(\mathrm{bb})$; a partial covering absorber with column density $N_{H}$ and covering factor $C$; a $6.4 \mathrm{keV}$ iron line; and a Compton reflection component ( $R=1$ for a $2 \pi$ solid angle).

\section{References}

de Martino D., Matt, G., Mukai, K. (+ 6 co-authors) 2001, A\&A, 377, 499 Haberl F. \& Motch C. 1995, A\&A 297, L37

Haberl F. 2002, in Physics of Cataclysmic Variables and Related Objects, ASP Conf. Ser., Vol. 261, p.151

Matt G. 1999, in "Annapolis Workshop on Magnetic Cataclysmic Variables", ASP Conf. Ser., Vol. 157, p. 299 